\title{
Stimulus set size and statistical coverage of the grammar in artificial grammar learning
}

\author{
Fenna H. Poletiek \\ Leiden University, Leiden, The Netherlands \\ AND \\ TeSSa J. P. van SCHIJNDEL \\ University of Amsterdam, Amsterdam, The Netherlands
}

\begin{abstract}
Adults and children acquire knowledge of the structure of their environment on the basis of repeated exposure to samples of structured stimuli. In the study of inductive learning, a straightforward issue is how much sample information is needed to learn the structure. The present study distinguishes between two measures for the amount of information in the sample: set size and the extent to which the set of exemplars statistically covers the underlying structure. In an artificial grammar learning experiment, learning was affected by the sample's statistical coverage of the grammar, but not by its mere size. Our result suggests an alternative explanation of the set size effects on learning found in previous studies (McAndrews \& Moscovitch, 1985; Meulemans \& Van der Linden, 1997), because, as we argue, set size was confounded with statistical coverage in these studies.
\end{abstract}

Learning the complex rules of language from linguistic stimuli is a striking example of inductive learning. Some researchers have emphasized the role of an innate human predisposition in natural grammar acquisition, with the environment playing a minor role (Chomsky, 1980; Pinker, 1994). Indeed, a major problem for cognitive learning explanations of grammar induction is that the stimulus sample contains too few exemplars to explain complete learning. However, a growing number of recent studies have suggested that structure induction relies, at least partly, on sensitivity to statistical properties of the structured environment (Chater \& Manning, 2006; Kuhl, 2004; Poletiek, 2006; Poletiek \& Chater, 2006; Poletiek \& Wolters, 2009; Redington, Chater, \& Finch, 1998). Here, we explore the hypothesis that statistical sample properties may be important for learning and, hence, compensate for limitations in number.

Although the problem of learning a structure with limited input is a core issue in linguistic theory and natural language acquisition research (Gold, 1967; Marcus, 1993), it has received little attention in statistical-learning accounts and experimental paradigms of grammar induction (Jamieson \& Mewhort, 2005). For example, only a few studies with the artificial grammar learning (AGL) paradigm, the experimental paradigm most often used to study implicit sequential structure learning, have addressed the question of how the number of exemplars in an input affects learning (McAndrews \& Moscovitch, 1985; Meulemans \& Van der Linden, 1997). Obviously, the most straightforward way to interpret the limited input problem is to look at the input sample's size. However, besides discrete sample information, statistical sample information about the grammar may be relevant to a learner as well. The purpose of the present study is to explore the influence of the amount of sample information about an underlying grammar — both discrete and statistical — on learning in an AGL task. We first will discuss previous experimental research on the effects of discrete input information. Next, we will introduce statistical coverage (SC) as a new statistical measure for the amount of sample information. Finally, we will report the effect of both measures when tested in an experiment.

In the AGL paradigm, participants are exposed to a number of exemplars (e.g., strings of letters) of a finite state structure during a training phase, without being informed about the structure. Next, in the test phase, the participants categorize new strings, half of which are grammatical and the other half ungrammatical. Performance in the test phase (proportion of correct categorizations) is taken as an indication of how much has been learned about the grammar at training. Moreover, response analyses can show whether categorizations were based on the actual grammaticality of the test items, fragment recognition (i.e., bigrams or trigrams), or memory for full exemplars (see Pothos, 2007, for a review).

McAndrews and Moscovitch (1985) used the AGL paradigm to investigate whether responses were based on memory for exemplars or knowledge of the grammar, after training with small and large input sets. They suggested that presentation with very few unique training exemplars

F. H. Poletiek, poletiek@fsw.leidenuniv.nl 
may induce memorization of individual exemplars, rather than structure learning, in the AGL task. Indeed, Vokey and Brooks (1992) reported exemplar-based learning with small input sets. With larger training sets, participants became increasingly sensitive to sequential structure in the stimuli, as McAndrews and Moscovitch showed. Thus, structure learning determined response after training with a relatively large number of exemplars, whereas exemplar memorization determined response after training with small sets. However, performance was not assessed separately in their study.

Building on McAndrews and Moscovitch's (1985) line of reasoning that grammar-based learning may be enhanced in large training sets, Meulemans and Van der Linden (1997) tested grammar learning versus fragment learning with both small (6 training exemplars) and large (125 training exemplars) sets. After training with numerous exemplars, participants showed more sensitivity to the grammaticality of test items, whereas fragment learning was observed after training with a few learning items. Although response sensitivity to item grammaticality is, of course, an indicator of how much about the grammar has been learned, no effect of sample size on performance was reported directly in the study by Meulemans and Van der Linden.

The results from the studies by McAndrews and Moscovitch (1985) and Meulemans and Van der Linden (1997) suggest that training with a large sample of exemplars enhances sensitivity to the grammaticality of new items, whereas training with a few exemplars induces learning through fragment knowledge or memory for exemplars. If item classification is a function of both analytic processing and item-specific information, item-specific information may be more salient in small stimulus sets (Regehr \& Brooks, 1993). Note, however, that a recent study failed to replicate the set size effect. Kinder and Assmann (2000) found no grammar-based responses in large and small set size conditions. Overall, the few experimental studies on the effect of stimulus set size on learning in AGL have offered inconclusive results. Hence, in the present experiment, we first explored the effect of input sample size on learning in AGL. Second, we investigated the possibility that SC of the grammar, rather than set size, might actually be responsible for variation in learning.

\section{Statistical Coverage}

Samples of exemplars of equal size can be shown to differ with regard to the amount of probabilistic information about the grammar they display. For example, a sample may contain a selection of either high-probability exemplars of the grammar or, alternately, exceptional and improbable items. Possibly, high-probability exemplars of a grammar tell more about that grammar than do low-probability ones. If so, grammar induction may be affected by variations in statistical sample information, and limitation in discrete information may be overcome by the richness of statistical information in the environment. It should be noted that previous statistical measures of exemplar information have been proposed to analyze grammaticality judgments on particular test items in the AGL task. However, the aim of these measures has been to model how statistical features of a particular input sample affect performance on a particular test set (Jamieson \& Mewhort, 2005; Pothos \& Bailey, 1999). To our knowledge, no previous attempts have been made thus far to model the amount of statistical information about the underlying grammar in the learning input.

The statistical information displayed in a sample of exemplars (which we call the statistical coverage of grammar $G$ by the sample) can be quantified using the probabilities of the individual exemplars to be generated by the grammar (Charniak, 1993; Poletiek, 2006; Poletiek \& Chater, 2006). Assume a finite state Markov grammar typically used in the AGL paradigm - that is, a network with paths and states producing strings of elements, by running from the first state to one of the output states along the paths. In probabilistic Markov structures (Charniak, 1993), probabilities are specified for the paths. Figure 1 displays the probabilistic Markov grammar $G$, first used by Meulemans and Van der Linden (1997), also used in the present study, with path probabilities specified.

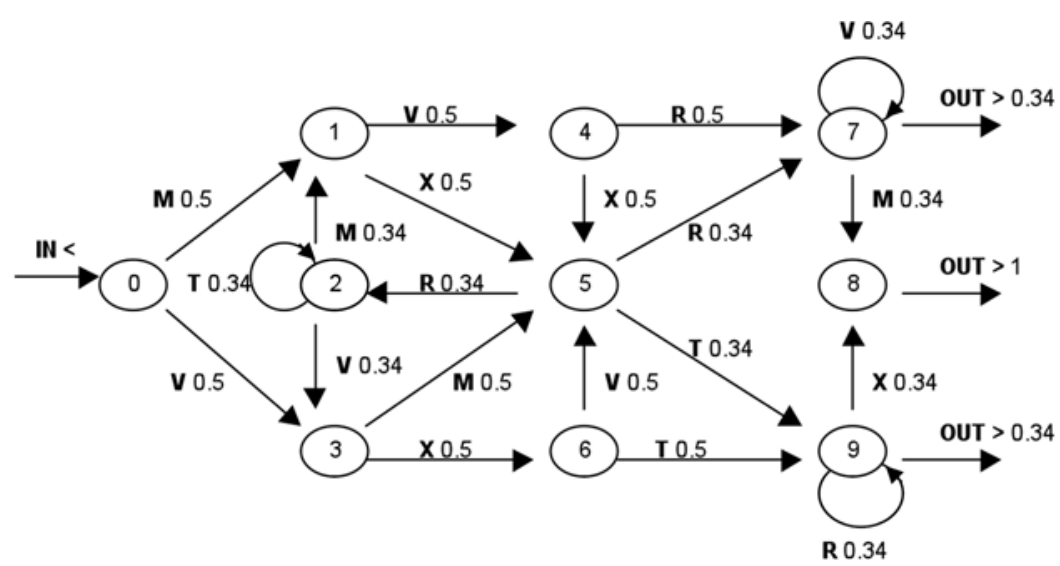

Figure 1. Finite state grammar $G$ used in a previous AGL study (Meulemans \& van der Linden, 1997) and the present one, with path probabilities specified. 
In the present example, these probabilities are unbiased. That is, all paths issuing from one state are equally probable.

The probability of an exemplar to be produced by $G$ is the product of the probabilities associated with the paths the exemplar runs through in the grammar (Charniak, 1993; Horning, 1969; Poletiek, 2006; Poletiek \& Chater, 2006; Van der Mude \& Walker, 1978).

For example, the probability of the exemplar MVRM is $p(\operatorname{MVRM} \mid G)=.5 \times .5 \times .5 \times .34=.0416$. Note that exemplar probability varies according to two characteristics: the values of its path probabilities and the exemplar's length. Indeed, since the probability of an exemplar is itself a product of probabilities, exemplar probability decreases with its length. In the present grammar $G$, probability and length of letter strings also correlate negatively, but not perfectly. Paths can have a probability of either .50 or .34 in $G$; a letter string made up of many .50 paths, may therefore be more probable than a shorter one having more .34 paths.

One important property of probabilistic Markov grammars is that the sum of the probabilities of all possible unique exemplars generated by the Markov grammar - the full language produced by $G$-is 1 . If a grammar generates an infinite number of unique exemplars (which is true for $G$, due to the loops in States 2, 7, and 9), the sum of all unique exemplar probabilities approximates 1 . Since the probabilities of the full set sum up to 1 , any subset's summed probability is a proportion: a value between 0 and 1 . This proportion represents the sample's SC of $G$, in the present proposal. In analogy, the proportion of discrete sample information in a subset of exemplars, as compared with the full output, would be the ratio of the number of unique exemplars in the sample to the total number of unique exemplars in the full language. Thus, two measures can be distinguished that describe the amount of information about the grammar in a sample of exemplars: set size and SC.

Note that SC and set size are naturally interdependent Adding new exemplars to a set will increase its SC, although not in a linear fashion: Adding high-probability items will result in a higher increase of SC than will adding low-probability items. The increase of discrete information is linear, however. It is equal for all types of exemplars (Charniak, 1993). In Figure 2, both the cumulative curve of the exemplar probabilities (i.e., the SC function) and the set size are displayed in one graph, for samples of exemplars of $G$.

Since the full output of $G$ is infinite, we assume it here, for practical reasons, to be finite with 300 unique exemplars.

On the $x$-axis, the unique exemplars of $G$ are numbered and arranged according to decreasing exemplar probabilities; every two points correspond to the sample of all exemplars between these points. The figure shows that for samples of highly probable exemplars, SC increases steeply, whereas low-probability exemplars add little to the sample's SC. Also, as can be seen in the figure, the sample of 40-50 most probable unique exemplars of $G$ makes about $15 \%$ of the total number of exemplars in the language, but it statistically covers about $70 \%$ of the language. As the sample size further increases, the difference between the discrete information and the SC decreases.

In previous AGL studies, only set size has been considered as a quantitative measure of sample information (McAndrews \& Moscovitch, 1985; Meulemans \& Van der Linden, 1997). Due to the natural correlation between SC

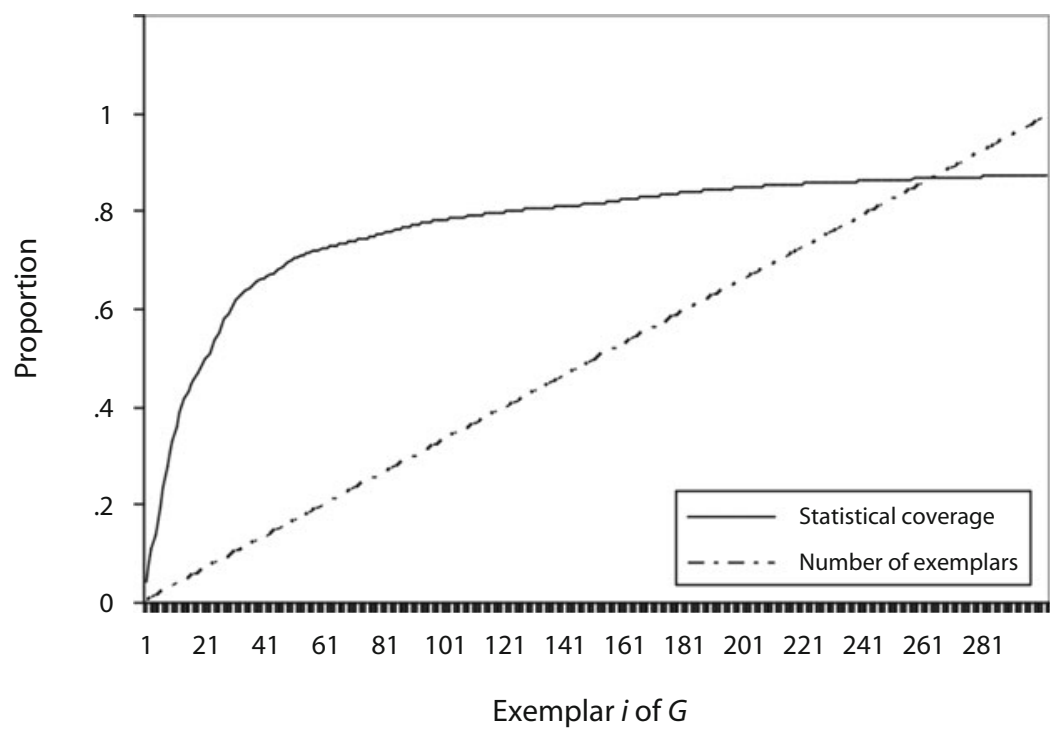

Figure 2. Proportion of statistical coverage of $G$ and proportion of total number of unique exemplars generated by $G$, for samples of the $i$ most probable exemplars of $G$. The exemplars are ranked on the $x$-axis in decreasing order of probability $p$ (exemplar $\mid G)$. In order to display the set size function-that is, the proportion of the number of exemplars in the sample to the total number of exemplars generated by $G$ - it is assumed that the output of $G$ is finite. 
Table 1

Mean Performance in All Conditions $(N=60)$

\begin{tabular}{|c|c|c|c|c|}
\hline \multirow{3}{*}{$\begin{array}{l}\text { Number of } \\
\text { Exemplars }\end{array}$} & \multicolumn{4}{|c|}{ Coverage } \\
\hline & \multicolumn{2}{|c|}{ Low $(n=30)$} & \multicolumn{2}{|c|}{$\operatorname{High}(n=30)$} \\
\hline & $M$ & $S D$ & $M$ & $S D$ \\
\hline Low & .54 & .11 & .60 & .08 \\
\hline High & .57 & .07 & .61 & .06 \\
\hline
\end{tabular}

and set size, these may have been confounded in these studies. We verified this confound in the materials of Meulemans and Van der Linden. SC was .06 for the small set condition (with 16 exemplars) and .70 for the large set condition (with 125 exemplars) (Experiments 1A and 1B). This suggests the possibility that $\mathrm{SC}$, rather than mere number of exemplars, explains that participants were more sensitive to the grammaticality of test items.

In the present experiment, set size and SC were manipulated orthogonally, and their effects on performance in AGL were tested independently. Furthermore, in line with previous studies on the set size effect, we explored whether the basis of performance (sensitivity to item grammaticality or fragment knowledge) would be affected by the two measures of sample information. Assuming that grammar induction relies, at least in part, on sensitivity to statistical aspects in the environment, we predicted that learning would be affected by the SC of the input sample.

\section{EXPERIMENT}

Four conditions were set up, with sets of exemplars varying orthogonally with regard to their size and statistical coverage. However, randomly manipulating SC and set size would tend to result in more short exemplars in high-SC samples than in the low-SC ones, because exemplar length is related to exemplar probability. To control for this, average exemplar length was matched for all the samples. In the test task, performance was assessed by the proportion of correct categorizations. If grammar induction is crucially affected by the sample's SC, rather than by its mere size, higher performance would be expected in the high-SC condition than in the low-SC condition, and no difference would be expected between large and small set sizes with equal SCs.

\section{Method}

Participants. Sixty university undergraduates ( 52 female, 8 male) participated in the experiment for course credit or payment. They were told that they were participating in a memory experiment.

Materials and Design. We used the finite state grammar $G$, displayed in Figure 1. Training set size and SC were manipulated between participants, in four conditions. Fifteen participants were assigned randomly to each condition. One group of participants was presented 30 exemplars (small set size group), and another group 60 exemplars (large set size group). For both small and large set groups, one stimulus set was made of exemplars with $\mathrm{SC}=.093$ (low-coverage group), and one with $\mathrm{SC}=.28$ (high-coverage group) (see Appendix A). Length of exemplars varied from 4 to 8 letters. All training sets contained at least two strings of each permissible length. The mean length of the exemplars was 6.4 letters for all conditions.

The test set of items was the same for all conditions (Appendix B). It contained 30 novel letter strings, half of which were gram- matical and half ungrammatical. The ungrammatical letter strings were constructed by switching the order of two successive letters of an exemplar. This procedure provided seven ungrammatical test items with an illegal bigram and eight items with legal bigrams in an illegal position. The first and last letters in a string were always permissible. Grammatical and ungrammatical strings in the test set were matched for length (see Appendix B).

Procedure. The experiment was administered in small groups of 3 participants on average. The participants faced a video screen on which the instructions and the stimuli were displayed. First, a block of four letter strings was displayed on the video screen for $15 \mathrm{sec}$. The participants were requested to "memorize as much as possible these letter strings." To ensure active processing of the stimuli, the participants were requested to write down the four strings after they had disappeared. Next, the same letter strings were displayed briefly again on the screen. This procedure was repeated until all the training stimuli had been presented. The training exemplars were presented in a predetermined random order.

Before starting the second part of the experiment, the test phase, the participants were informed that the strings were made according to some rule structure. They were handed out an answer form and were asked to judge the grammaticality of 30 new letter strings. They were informed that half of the test strings were in accordance with these rules.

\section{Results}

Performance was calculated by the proportion of correct classifications. Performance in all the conditions is displayed in Table 1.

First, an ANOVA on performance, with number of exemplars (set size: high or low) and SC (high or low) as between-subjects variables, showed a significant main effect of coverage $[F(1,56)=6.1, p=.016]$. No main effect of set size $[F(1,56)=0.63, p>.05]$ and no interaction effects $[F(1,56)=0.48, p>.05]$ were found.

Second, we tested whether SC also affected sensitivity to item grammaticality and to fragment patterns. For this purpose, we performed a simultaneous regression analysis with test item grammaticality, test item global associative chunk strength (GACS), and anchor associative chunk strength (AACS) as variables predicting endorsement of each test item (for a similar analysis, see Reber \& Perruchet, 2003). GACS and AACS were calculated for each test item for each condition separately, according to Knowlton and Squire's (1994) method. GACS of a test item is the average frequency with which its fragments (bigrams and trigrams) occurred in the training sample. AACS is the average of an item's bigram and trigram frequencies occurring in anchor positions (at the beginning and the end of training exemplars). Together, sensitivity to GACS and AACS indicates fragment-based learning. The GACS and AACS of each individual test item differed per

Table 2

Summary of Simultaneous Regression Analysis for Variables

Predicting Endorsement of Test Items for Low and High Statistical Coverage (SC) Conditions

\begin{tabular}{lcc}
\hline & \multicolumn{2}{c}{$\beta$} \\
\cline { 2 - 3 } Variable & Low SC & High SC \\
\hline Grammaticality & .21 & $.41^{* *}$ \\
Global ACS & .11 & .07 \\
Anchor ACS & .17 & .11 \\
\hline
\end{tabular}

Note-ACS, associative chunk strength. ${ }^{* *} p<.01$. 
condition, because the training sets differed and, hence, the frequency distribution of bigrams and trigrams.

The correlation between the predictors indicating fragment-based learning (GACS and AACS of the test items) was $r=.69$. Correlations between the predictor item of grammaticality and the fragment-learning measures were low (grammaticality-GACS, $r=.38$; grammaticality-AACS, $r=.18$ ). In Table 2 , the standardized weights are displayed for both SC groups. Grammaticality predicted endorsement for high-coverage learning sets only. Fragment-learning measures did not predict endorsement in any group. The same regression analysis was done for the two set size groups, which performed equally well on accuracy. No difference in basis of response for large and small sets was found. Both conditions relied equally on grammar knowledge ( $\beta=.26, p=.05$, for small sets and .31, $p=.03$, for high sets) and on GACS and AACS (all $\beta$ s being nonsignificant).

We compared sensitivity to grammaticality and to ACS between SC conditions. A significant effect of SC on the weight of grammaticality was found $[F(2,113)=6.7$, $p=.002]$, indicating that responses were based on grammaticality more often in the high-SC condition than in the low-SC condition. No difference between the weights of GACS and AACS for low and high SC was found. This analysis suggests that better performance in AGL after training with samples providing a large SC of the grammar may relate to enhanced sensitivity to the underlying structure of the stimuli under this condition.

\section{Discussion}

Our data suggest that performance in an AGL task is crucially facilitated by exposure to input samples containing many highly probable exemplars of the grammar, rather than just many exemplars. When SC (the summed probability of the sample's exemplars) was separated from set size, SC affected learning, whereas set size did not. Specifically, learning after training with a high-SC sample was characterized by higher sensitivity to item grammaticality than was learning with a low-SC sample. As a result, our analysis suggests that previously reported effects of set size in AGL might actually have been due to SC variation that was confounded with set size (Meulemans \& Van der Linden, 1997).

The SC effect has interesting parallels in domains outside AGL. For example, the false memory effect, the illusion that a word has been studied (Roediger \& McDermott, 1995), has been shown to depend on the forward association strength between this critical thematic word (sleep) not presented and the list of related words (e.g., bed, tired, yawn) participants studied (Brainerd \& Wright, 2005). The association strength is the probability that list items will be elicited as a response to the critical word in a separate association task. The association strength between the underlying thematic word (falsely recalled) and the actually studied words in the false memory paradigm is an analogue to the probability that training exemplars will be produced by the grammar in AGL. Although in earlier false memory research, set size (studied words) was reported to facilitate inducing the critical word (Roediger \& McDermott, 1995), recent research suggests that it is not the mere number of items in the list studied that crucially activates the underlying critical item, but the summed association probabilities (in analogy, the SC) between the studied items and the critical item (Alonso, Diez, \& Fernandez, 2007; Robinson \& Roediger, 1997). This finding narrowly parallels the present one in the AGL domain, suggesting that both grammar induction and false memory depend on the statistical relation between the input stimuli to the underlying theme or structure.

SC variations may also play a role in natural grammar learning. Children acquire full mastering of their natural grammar after exposure to a small sample of the full language only. Child-directed speech research suggests that the content of this early sample deviates from adult linguistic samples: It includes, among other times, more frequent syntactical constructions than does adult speech (Philips, 1973; Pine, 1994; Snow, 1972). This obviously resembles the high-coverage condition. Hence, the high SC of samples of speech directed at children may boost the grammar-learning process in the early stage of learning. For that matter, our results underscore Newport's (1990) finding that in tasks involving structure induction, more is not always better. Instead, SC of the structure by the input sample, rather than mere sample size, seems to be an important contributor to the inductive learnability of the structure underlying that input.

\section{AUTHOR NOTE}

This research was supported by a grant from the Netherlands Organization for Scientific Research. We thank Jarry Porsius for his help with the data analyses. Correspondence concerning this article may be sent to F. H. Poletiek, Institute of Psychology, Leiden University, P.O. Box 9555, 2300RB Leiden, The Netherlands (e-mail: poletiek@ffsw.leidenuniv.nl).

\section{REFERENCES}

Alonso, M. A., Diez, E., \& Fernandez, A. (2007, November). Effects of backward associative strength on the false recognition of words. Poster presented at the 48th Annual Meeting of the Psychonomic Society, Long Beach, CA.

Brainerd, C. J., \& Wright, R. (2005). Forward association, backward association, and the false-memory illusion. Journal of Experimental Psychology: Learning, Memory, \& Cognition, 31, 554-567.

Charniak, E. (1993). Statistical language learning. Cambridge, MA: MIT Press.

Chater, N., \& Manning, C. D. (2006). Probabilistic models of language processing and acquisition. Trends in Cognitive Sciences, 10, 335-344.

CHOMsky, N. (1980). Rules and representations. New York: Columbia University Press.

GoLD, E. M. (1967). Language identification in the limit. Information \& Control, 10, 447-474.

Horning, J. J. (1969). A study of grammatical inference (Tech. Rep. CS 139). Stanford, CA: Stanford University, Computer Science Department.

Jamieson, R. K., \& Mewhort, D. J. K. (2005). The influence of grammatical, local, and organizational redundancy on implicit learning: An analysis using information theory. Journal of Experimental Psychology: Learning, Memory, \& Cognition, 31, 9-23.

Kinder, A., \& Assmann, A. (2000). Learning artificial grammars: No evidence for the acquisition of rules. Memory \& Cognition, 28, 13211332. 
Knowlton, B. J., \& SQuire, L. R. (1994). The information acquired during artificial grammar learning. Journal of Experimental Psychology: Learning, Memory, \& Cognition, 20, 79-91.

Kunt, P. K. (2004). Early language acquisition: Cracking the speech code. Nature Reviews Neuroscience, 5, 831-843.

Marcus, G. F. (1993). Negative evidence in language acquisition. Cognition, 46, 53-85.

McAndrews, M. P., \& Moscovitch, M. (1985). Rule-based and exemplar-based classification in artificial grammar learning. Memory \& Cognition, 13, 469-475.

Meulemans, T., \& Van der Linden, M. (1997). Associative chunk strength in artificial grammar learning. Journal of Experimental Psychology: Learning, Memory, \& Cognition, 23, 1007-1028.

NEwPORT, E. L. (1990). Maturational constraints on language learning. Cognitive Science, 14, 11-28.

PhiLIPS, J. R. (1973). Syntax and vocabulary of mothers' speech to young children: Age and sex comparisons. Child Development, 44, 182-185.

Pine, J. M. (1994). The language of primary caregivers. In C. Gallaway $\&$ B. J. Richards (Eds.), Input and interaction in language acquisition (pp. 15-37). Cambridge: Cambridge University Press.

PINKER, S. (1994). The language instinct. Harmondsworth, U.K.: Penguin.

Poletiek, F. H. (2006). Natural sampling of stimuli in (artificial) grammar learning. In K. Fiedler \& P. Juslin (Eds.), Information sampling and adaptive cognition (pp. 440-455). Cambridge: Cambridge University Press.

Poletiek, F. H., \& Chater, N. (2006). Grammar induction profits from representative stimulus sampling. In R. Sun \& N. Miyake (Eds.), Proceedings of the 28th Annual Conference of the Cognitive Science Society (pp. 1968-1973). Mahwah, NJ: Erlbaum.
Poletiek, F. H., \& Wolters, G. (2009). What is learned about fragments in artificial grammar learning? A transitional probabilities approach. Quarterly Journal of Experimental Psychology, 62, 868-876.

Pothos, E. M. (2007). Theories of artificial grammar learning. Psychological Bulletin, 133, 227-244.

Pothos, E. M., \& Bailey, T. M. (1999). An entropy model of artificial grammar learning. In Proceedings of the Twenty-First Annual Conference of the Cognitive Science Society (pp. 549-554). Mahwah, NJ: Erlbaum.

Reber, R., \& Perruchet, P. (2003). The use of control groups in artificial grammar learning. Quarterly Journal of Experimental Psychology, 56A, 97-115.

Redington, M., Chater, N., \& Finch, S. (1998). Distributional information: A powerful cue for acquiring syntactic categories. Cognitive Science, 22, 425-469.

Regehr, G., \& BRoOKs, L. R. (1993). Perceptual manifestations of an analytic structure: The priority of holistic individuation. Journal of Experimental Psychology: General, 122, 92-114.

Robinson, K. J., \& RoEdiger, H. L., III (1997). Associative processes in false recall and false recognition. Psychological Science, 8, 231-237.

Roediger, H. L., III, \& McDermott, K. B. (1995). Creating false memories: Remembering words not presented on lists. Journal of Experimental Psychology: Learning, Memory, \& Cognition, 21, 803-814.

SNow, C. E. (1972). Mothers' speech to children learning language. Child Development, 43, 549-565.

VAN DER Mude, A., \& Walker, A. (1978). On the inference of stochastic regular grammars. Information \& Control, 38, 310-329.

VoKeY, J. R., \& BROOKS, L. R. (1992). The salience of item knowledge in learning artificial grammars. Journal of Experimental Psychology: Learning, Memory \& Cognition, 18, 328-344.

APPENDIXA

Training Stimuli With $p$ (stimulus $\mid G)$, for All Conditions

\begin{tabular}{|c|c|c|c|c|c|}
\hline \multicolumn{4}{|c|}{$\begin{array}{c}\text { Large Set and Low } \\
\text { Statistical Coverage Condition }\end{array}$} & \multicolumn{2}{|c|}{$\begin{array}{c}\text { Small Set and Low } \\
\text { Statistical Coverage Condition }\end{array}$} \\
\hline Probability & Exemplar & Probability & Exemplar & Probability & Exemplar \\
\hline .00926 & MXRV & .00051 & MVRVVVV & .02778 & MXRM \\
\hline .00926 & MXTR & .00051 & MVXRVVV & .02778 & MXTX \\
\hline .00926 & VMRV & .00051 & MVXTRRR & .01389 & VXVTX \\
\hline .00926 & VMTR & .00051 & MXRMXRV & .00463 & VXVRV \\
\hline .00463 & VXVTR & .00051 & MXRMXTR & .00154 & VMRVMR \\
\hline .00309 & MXTRR & .00051 & MXRTMXR & .00154 & VMRVMT \\
\hline .00309 & VMRVV & .00051 & MXRTMXT & .00154 & VXTRRR \\
\hline .00309 & VMTRR & .00051 & MXRTVMR & .00154 & VXVRVV \\
\hline .00154 & MVRVVV & .00051 & MXRTVMT & .00154 & VXVTRR \\
\hline .00154 & MVXRVV & .00051 & MXRVMRV & .00103 & MXRVVV \\
\hline .00154 & MVXTRR & .00051 & MXRVMTR & .00103 & MXTRRR \\
\hline .00154 & MXRMXR & .00051 & VMRMXRV & .00103 & VMRVVV \\
\hline .00154 & MXRMXT & .00051 & VMRMXTR & .00103 & VMTRRR \\
\hline .00154 & MXRVMR & .00051 & VMRTMXR & .00051 & VMRMXRV \\
\hline .00154 & MXRVMT & .00051 & VMRTMXT & .00051 & VMRMXTR \\
\hline .00154 & VMRMXR & .00051 & VMRTVMR & .00051 & VMRTMXR \\
\hline .00154 & VMRMXT & .00051 & VMRTVMT & .00051 & VMRTMXT \\
\hline .00154 & VMRVMR & .00051 & VMRVMRV & .00051 & VMRTVMR \\
\hline .00154 & VMRVMT & .00051 & VMRVMTR & .00051 & VMRTVMT \\
\hline .00154 & VXTRRR & .00051 & VXTRRRR & .00051 & VMRVMRV \\
\hline .00154 & VXVRVV & .00051 & VXVRVVV & .00051 & VMRVMTR \\
\hline .00154 & VXVTRR & .00051 & VXVTRRR & .00051 & VXTRRRR \\
\hline .00103 & MXRVVV & .00034 & MXRVVVV & .00051 & VXVRVVV \\
\hline .00103 & MXTRRR & .00034 & MXTRRRR & .00051 & VXVTRRR \\
\hline .00103 & VMRVVV & .00034 & VMRVVVV & .00034 & MXRVVVV \\
\hline .00103 & VMTRRR & .00034 & VMTRRRR & .00034 & MXTRRRR \\
\hline .00077 & VXVRMXR & .00011 & MXRVVVVV & .00034 & VMRVVVV \\
\hline .00077 & VXVRMXT & .00011 & MXTRRRRR & .00034 & VMTRRRR \\
\hline .00077 & VXVRVMR & .00011 & VMRVVVVV & .00011 & VMRVVVVV \\
\hline .00077 & VXVRVMT & .00011 & VMTRRRRR & .00011 & VMTRRRRR \\
\hline
\end{tabular}


APPENDIX A (Continued)

\begin{tabular}{|c|c|c|c|c|c|}
\hline \multicolumn{4}{|c|}{$\begin{array}{c}\text { Large Set and High } \\
\text { Statistical Coverage Condition }\end{array}$} & \multicolumn{2}{|c|}{$\begin{array}{l}\text { Small Set and High } \\
\text { Statistical Coverage Condition }\end{array}$} \\
\hline Probability & Exemplar & Probability & Exemplar & Probability & Exemplar \\
\hline .02778 & MXTX & .00034 & VMRVVVV & .04167 & MVRM \\
\hline .02778 & VMRM & .00034 & VMTRRRR & .04167 & VXTX \\
\hline .01389 & MVRVM & .00017 & MXRTMXTR & .02778 & MXRM \\
\hline .01389 & MVXRM & .00017 & MXRTTMXR & .02778 & MXTX \\
\hline .01389 & MVXT & .00017 & MXRTTMXT & .02778 & VMRM \\
\hline .01389 & MVXTX & .00017 & MXRTTVMR & .01389 & MVRVM \\
\hline .01389 & VXTR & .00017 & MXRTTVMT & .01389 & MVXRM \\
\hline .01389 & VXTRX & .00017 & MXRTVMRV & .01389 & MVXTX \\
\hline .01389 & VXVR & .00017 & MXRTVMTR & .01389 & VXTRX \\
\hline .01389 & VXVRM & .00017 & MXRVMRVV & .01389 & VXVRM \\
\hline .01389 & VXVT & .00017 & MXRVMTRR & .01389 & VXVTX \\
\hline .01389 & VXVTX & .00017 & VMRMXRVV & .00926 & MXRVM \\
\hline .00926 & MXRV & .00017 & VMRMXTRR & .00463 & MVRVVM \\
\hline .00926 & MXRVM & .00017 & VMRTMXRV & .00463 & MVXRVM \\
\hline .00926 & MXTR & .00017 & VMRTMXTR & .00231 & MXRMVRM \\
\hline .00926 & VMRV & .00017 & VMRTTMXR & .00231 & MXRVXTX \\
\hline .00926 & VMTR & .0 & $\mathrm{VMR}^{\top}$ & .00116 & MVXRMVRM \\
\hline .00926 & VMTRX & .0 & VMRTTVMR & .00116 & MVXRVXTX \\
\hline .00463 & MVRV & & VMRT & .00116 & VXVRMVRM \\
\hline .00463 & MVXRV & .00017 & VMRTVMRV & .00116 & VXVRVXTX \\
\hline .00463 & MVXTR & & VMRTVMTR & .00077 & MVXRMXRM \\
\hline .00463 & VXTRR & .00017 & VMRVMRVV & .00077 & MVXRMXTX \\
\hline .00463 & VXVRV & .00017 & VMRVMTRR & .00077 & MVXRVMRM \\
\hline .00309 & MXTRR & .00017 & VXTRRRRR & .00077 & MVXRVMTX \\
\hline & MXRVVI & & VXVRVVVV & .00077 & MXRMVRVM \\
\hline .00103 & MXTRRR & .00017 & VXVTRRRR & .00077 & MXRMVXRM \\
\hline .00103 & VMRVVV & .00011 & MXRVVVVV & .00077 & MXRMVXTX \\
\hline .00103 & VMTRRR & .00011 & MXTRRRRR & .00077 & MXRTMVRM \\
\hline .00034 & MXRVVVV & .00011 & VMRVVVVV & .00077 & MXRTVXTX \\
\hline .00034 & MXTRRRR & .00011 & VMTRRRRR & .00077 & MXRVXTRX \\
\hline
\end{tabular}

APPENDIX B

Test Items

\begin{tabular}{ll}
\hline Grammatical & Ungrammatical \\
\hline MVRV & MXVT \\
MVXR & VRMM \\
VMTX & VTXX \\
MXRVV & MVVRM \\
MXTRX & VXRTR \\
VMRVM & VXTVX \\
MVXTRX & MXRXMT \\
VXTRRX & VMRXMT \\
VXVRVM & VXVVRV \\
MVRVVVM & MXRVTXX \\
VMRMVRM & VMMRXRV \\
VMRVXTX & VMRMTXR \\
MXRVXVRM & MVRXMVRM \\
MXRVXVTX & MXRVTMRR \\
VMRMVRVM & VRMVVVVV \\
\hline
\end{tabular}

(Manuscript received March 11, 2009;

revision accepted for publication July 7, 2009.) 